\title{
Adrian MacKenzie (2017) Archaeology of a Data Practice. Cambridge, MA: The MIT Press. 252 pages. ISBN: 978-1-5179-0064-9
}

\author{
Martina Klausner \\ martina.klausner@hu-berlin.de
}

How can critical thought come to terms with knowledge practices fundamentally shaped by machine learning? What might critical thinkers learn from machine learners? And how could critical thought itself be transformed by engaging with digital data analytics? As machine learning the programming of computers to learn from data - has spread across various domains and shapes knowledge practices in various fields, these questions are timely and pressing. To explore them, Adrian Mackenzie's pathway led him into learning machine learning: Combing through the vast corpus of machine learning literature, manuals and tutorials, using machine learning methods to analyse the references to machine learning in scientific literature, attending to the histories of some machine learners and following the proliferation and spread of others across various domains of knowledge, Mackenzie literally enacts what it entails to learn machine learning. While the subtitle of the book calls this approach an archaeology of a data practice, Mackenzie is not only digging deep into genealogies and ancestries of contemporary data methods, devices and infrastructures, but also dissects the very texture of machine learning and then weaves the various threads back together. Through this, he moves the reader through the central elements of machine learning that make up the seven empirical chapters of the book. To state this clearly at the beginning: large parts of the book are not an easy read (unless you are Paul Dourish maybe, who claims to have read the book on a flight from Los Angeles to
Sydney). The book is populated with codes, tables, equations, graphic plots, list of references and is impressing with the synchronicity of engaging in the very practices that are observed. Attending to (seven) central facets of machine learning and various machine learners (human and non-human) Mackenzie carefully excavates some of the larger transformations and also smaller shifts that have emerged over the last decades in computer science, statistics, engineering and other scientific domains and especially across them.

The book left me puzzled in various ways. First and foremost, I was deeply impressed by the serious and deep engagement with the insides, technicalities and details of machine learning. Reading rather felt like a learning process itself where the teacher doesn't give you the answers but asks you to move along and explore yourself. In a sense, the book announces a beginning, an opening up of questions to examine further. It's the book you need to read to get an idea what questions to ask machine learners, assuming you want to go beyond familiar tropes of reductionism, lack of control and transparency, the risks of automation of knowledge production through machine learning. Those issues are still important and necessarily addressed, however Mackenzie gives us a clearer (yet more complicated) idea of where and how transformations are emerging and should be attended. Citing Foucault, Mackenzie insists, that "knowing the conditions, setting out the rules, and identifying the relations that striate the density and complexity of practice is a precon- 
dition to any transformation in practice" (p. 217). And Mackenzie does exactly this, examining those preconditions.

The chapter Diagramming Machines, for example, unfolds the landscape of machine learning and thereby redraws the very frame of analysis. One of the sweeping developments in fields of machine learning has been the expansion and migration of devices and methods into various domains of science and industry. It is the diagrammatic formation of machine learners, their intersecting references and migratory pattern (beyond the common focus on "the algorithm"), which should catch our attention. With the diagram, which basically refers to a graphic description of abstraction in space, Mackenzie refers to both Foucault and Deleuze. As he explains in the glossary of the book "the diagram is a form of abstractions concerned with functioning and operations. In Gilles Deleuze's reading of Michel Foucault, diagrams display relations of force and construct models of truth" (p. 220) Most importantly, machine learners do not convey meaning in themselves (they are "a-signifying" in Deleuze's and Guattari's words), but generate ideas through bringing diverse fields into relation. A later chapter, Regularizing and Materializing Objects, convincingly illustrates the diagramming effects with the case of genome research: the scientific hyperobject "the genome" materializes and stabilizes through the specific entanglement of genetics, bioinformatics and machine learning, their data infrastructures, methods and devices, in unprecedented ways. Another central theme, which Mackenzie touches upon in different variations, is the question of abstraction and formalization generated by machine learning. While reduction and prediction might be central effects of processing data with machine learning methods, Mackenzie foregrounds multiplication and concretization. Much of the actual "learning" of machine learning, Mackenzie show us, is a constant, experimental effort in an unique entanglement of operation and observation. All along, Mackenzie's style is modest and cautious in the way he carefully points to transformations, never lapsing into polemic statements and hypes.

At the same time, and actually even for the same reasons, Mackenzie's Machine Learners also left me puzzled with a sense of frustration. The firm and detailed grounding of the analysis in the practices of machine learners is at times not only hard to comprehend and digest, but also left me with a strange feeling of boundedness, despite the many traces and movements outlined. In a sympathetic reading, I consider this a form of re-enactment of machine learners: their awkward indifference to the concrete settings where data are produced, the detachment of data from their messy groundings, the almost hermetic focus on inward processes of operation, observation and optimization, the seemingly indiscernible, tightly interwoven texture of links, iterations, expansion and trimming, regardless if the data at hand concern breast cancer research or the detection of cat images on the internet.

This analytic mode of re-enactment relates more generally to issues of immersion and intervention, of proximity and partiality with(in) research fields, which increasingly gain attention in STS and ethnographic research. In the preface, with the very first sentence, Mackenzie states, that even though "this book is not an ethnography, it has an ethnographic situation" (p. xi). However, in my understanding, the most powerful ethnographic effect is that it consists of something fundamentally more than a situation, it creates relations: in a way, it is always a distortion of a situation and thereby renders visible how things could be otherwise. There are und must be different ways to create such relations and irritations. Take Annelise Riles' twisting of The Network Inside Out (2001), where the network (of international human rights activists) was both her research object and she was an active part of it. How to study something which we are fundamentally part of? Another example, thematically closer to Mackenzie's book: Christopher Kelty's Two Bits (2008), which is simultaneously investigating and engaging with Free Software practices. In some ways Kelty's, Mackenzie's, and Riles' work are similar as they embrace proximity and immersion as the very strength of their analysis. Taking seriously the aesthetics of practices as object of analysis while taking part in it. Writing about code while writing code. However, Riles for example does purposely juxtapose and irritate this familiarity and thereby makes the contours 
of the network appear in a figure-ground twist. What would a figure-ground twist of machine learners be? Or more generally, how could such an examination and description irritate and thereby create new relations? Mackenzie hints at some potentials for critical thought: we should ask how "divergence remains possible" (p. 102) despite the massive regularization and formalization of knowledge and how we might learn to experiment with prediction in the endless relays of reference provided by machine learning in a different way. Yet, those potentials remain mostly vague and almost vanish in light of the diagramming machinery. As stated above, the book's special feature is the generation of questions and potential pathways to be further explored. Overall, it provides supervised machine learning for critical thinkers, to enable them "to diagram a diagrammatic domain", as Mackenzie calls it (p. 207). As science, governance, industry, and many other domains are already fundamentally shaped by data practices, seeking for and tinkering with alternative ways of relating machine learners with elements of critical thought seems indispensable.

Kelty C M (2008) Two Bits: The cultural significance of free software. Duke University Press.

Riles A (2001) The Network Inside Out. University of Michigan Press. 